\title{
Small-Kernel Super-Resolution Methods for Microscanning Imaging Systems
}

\author{
Jiazheng Shi, ${ }^{*}$ Stephen E. Reichenbach, ${ }^{*}$ and James D. Howe ${ }^{\dagger}$ \\ * Computer Science and Engineering Department, \\ University of Nebraska - Lincoln \\ ${ }^{\dagger}$ Night Vision and Electronic Sensors Directorate, U.S. Army
}

This paper presents two computationally efficient methods for superresolution reconstruction and restoration for microscanning imaging systems. Microscanning creates multiple low-resolution images with slightly varying sample-scene phase shifts. The digital processing methods developed in this paper combine the low-resolution images to produce an image with higher pixel resolution (i.e., super-resolution) and higher fidelity. The methods implement reconstruction to increase resolution and restoration to improve fidelity in one-pass convolution with a small kernel. One method is a smallkernel Wiener filter and the other method is a parametric cubic convolution filter (designated 2D-5PCC-R). Both methods are based on an end-to-end, continuous-discrete-continuous (CDC) microscanning imaging system model. Because the filters are constrained to small spatial kernels they can be efficiently applied by convolution and are amenable to adaptive processing and to parallel processing. Experimental results with simulated imaging and with real microscanned images indicate that the small-kernel methods efficiently and effectively increase resolution and fidelity. (c) 2005 Optical Society of America

OCIS codes: $100.6640,100.3020,100.2000$

\section{Introduction}

Improvements in the resolution and fidelity of digital imaging systems have substantial value for remote sensing, military surveillance, and other applications, especially those for which a large field-of-view is desirable and the distance to objects of interest cannot be reduced. Advances in optics and sensor technologies offer one path for increasing the spatial resolution and fidelity of imaging systems, but such hardware improvements can be costly and/or 
limited by physical constraints. ${ }^{1,2}$ Digital image processing offers an alternative path for improving image quality.

Digital super-resolution reconstruction and restoration methods can substantially increase resolution and fidelity of an imaging system. Reconstruction methods increase pixel resolution beyond that of the physical imaging system by estimating values on a finer grid or lattice. Restoration methods increase fidelity beyond that of the physical imaging system by correcting for acquisition artifacts such as blurring, aliasing, and noise. Super-resolution reconstruction and restoration can be combined to produce images with greater resolution and higher fidelity. ${ }^{3}$

Typically, super-resolution methods produce an enhanced image from multiple lowresolution images with either different sample-scene phase or with different blurring functions. ${ }^{4,5}$ Microscanning is a systematic approach for acquiring images with slightly differing sample-scene phase - between successive images, the system is shifted slightly, either in a predetermined pattern or in a random pattern. As in most super-resolution methods, the methods developed in this paper presume global sample-scene phase shifts.

Most super-resolution methods can be decomposed into three tasks: registration, reconstruction, and restoration. These tasks can be implemented as separate steps or in one or two combined steps.

- Registration is the process of orienting several images of a common scene with reference to a single coordinate system. Registering images to subpixel accuracy is a crucial factor that greatly impacts super-resolution processing. Systems with precisely controlled phase-shifting may be trivial to register. Popular methods for subpixel image registration include phase or cross correlation registration ${ }^{6,7}$ and gradient-based registration. 8,9

- Image reconstruction is the process of estimating the image value at arbitrary locations on a spatial continuum from a set of discrete samples. For super-resolution imaging, the aim of reconstruction is to produce an image with pixels on a high-resolution grid with uniform spacing from the multiple, registered images which may have pixels that are irregularly distributed. Popular methods for reconstruction include nearest neighbor interpolation, bi-linear interpolation, cubic-spline interpolation, piecewise cubic convolution, and cubic optimal maximal-order-minimal-support (o-Moms) interpolation. ${ }^{10}$ Kriging, which originated in the geostatistical community, is a popular method for interpolating spatial data. ${ }^{11,12}$

- Image restoration is the process of recovering a more accurate image of a scene by correcting or reducing degradations such as acquisition blurring, aliasing, and noise. ${ }^{13}$ Basic methods for image restoration include deconvolution, least-squares filters, and 
iterative approaches. $^{14}$

Digital processing for super-resolution is an area of active research. ${ }^{1,15}$ Approaches based on non-uniform interpolation are the most intuitive. Computational efficiency is their major advantage. For example, Alam et al. proposed a super-resolution method for infrared images, using a gradient-based method to estimate shift, the weighted nearest-neighbor approach to align irregularly displaced low-resolution images to form a super-resolution image, and finally the Wiener filter for image restoration. ${ }^{9}$ However, nonuniform interpolation approaches do not account for varying acquisition conditions for the low-resolution images nor guarantee the optimality of the end-to-end imaging system. ${ }^{3}$ Tsai and Huang investigated super-resolution imaging in frequency domain, ${ }^{16}$ using the shift theorem of the Fourier transform and the aliasing relationship between low-resolution images and the ideal super-resolution image. High frequency components are extracted from aliased low frequency components. Kim et al. extended this approach for blurred and noisy images and developed a weighted recursive least-squares algorithm. ${ }^{17,18}$ Unfortunately, Fourier-domain methods are computational costly. Super-resolution methods based on stochastic theories employ a priori knowledge about a scene and noise. For example, Shultz and Stevenson ${ }^{19}$ proposed a Bayesian restoration with a discontinuity-preserving prior image model and Elad et al. ${ }^{5,20}$ approached superresolution by unifying the maximum likelihood (ML), maximum a posteriori (MAP), and projection onto convex set (POCS). Stochastic approaches also may have high computational complexity and sub-optimality for the end-to-end imaging system. Computationally efficient spatial-domain methods have been proposed. Nguyen et al. ${ }^{21}$ proposed efficient block circulant preconditioners for solving the Tikhonov regularized superresolution problems by using the conjugate gradient method, but the method requires perfect shift estimation. ${ }^{22}$ Farsiu et al. proposed a robust method that defines the cost function of regularization using the $L_{1}$ norm minimization. ${ }^{23}$ The method is robust for estimation errors of shift and noise, but the system model accounts for only acquisition in the end-to-end system and the proposed fast solution is iterative. Even though interpolation and restoration are done simultaneously, computational costs for real-time applications are a concern with iterative methods.

This paper investigates super-resolution methods for microscanning imaging systems that can be implemented efficiently with small convolution kernels. The approach is based on an end-to-end, CDC system model that accounts for the fundamental tradeoff in imaging system design between acquisition blurring related to optics, detectors, and analog circuits and aliasing due to sampling. The system model also accounts for noise associated with unpredictable signal and system variations and quantization, for mis-registration of the lowresolution images, and for the display system. The approach uses one-pass convolution with small kernels for computationally efficient reconstruction and restoration. This approach also is amenable to adaptive processing and to parallel processing with appropriate hardware 
support.

The rest of this paper is organized as follows. Section 2 presents the CDC system model, introduces microscanning, and formulates the system fidelity. Section 3 derives super-resolution reconstruction and restoration filters, including the optimal Wiener filter, a spatially constrained Wiener kernel, and a parametric cubic convolution kernel. Section 4 presents experimental results for images from a simulation and from a real imaging system. Section 5 summarizes this paper and describes issues for future work.

\section{System Model and Problem Formulation}

This section presents the CDC system model, describes microscanning, and formulates the fidelity of a microscanning imaging system based on the system model. The system model is introduced in the spatial domain of the image, but the problem and fidelity analysis are formulated in the Fourier frequency domain so that spatial convolution can be considered as pointwise multiplication of transform coefficients.

\section{A. Continuous-discrete-continuous system model}

The super-resolution methods developed in this paper are based on the CDC model pictured in Fig. 1. This imaging system model is relatively simple, yet captures the most significant degradations in typical imaging systems: linear shift-invariant blurring, characterized by the acquisition point spread function (PSF) $h$; aliasing, due to sampling a continuous function on a uniform, rectangular lattice $\Perp$; additive system noise $e$; and display, characterized by the display PSF $d$.

With this model, a single low-resolution digital image $p$ is defined mathematically as:

$$
p[m, n]=\iint s(x, y) h(m-x, n-y) d x d y+e[m, n],
$$

where $[m, n]$ are integer pixel indices for the digital image $p$ and $(x, y)$ are continuous coordinates for the scene $s$. For notational convenience, and without loss of generality, the spatial coordinates are normalized in units of the sampling interval. In practice, the spatial extent of the image is finite, but that issue is not significant for the following analyses.

Restoration and reconstruction can be implemented in one step by convolving the image $p$ with the filter PSF $f$ to produce a processed image of arbitrary resolution, which can then be displayed. Modeling display as a linear shift-invariant process characterized by the display PSF $d$, the resulting continuous output image $r$ is:

$$
r(x, y)=\iint\left(\sum_{m} \sum_{n} p[m, n] f\left(x^{\prime}-m, y^{\prime}-n\right)\right) d\left(x-x^{\prime}, y-y^{\prime}\right) d x^{\prime} d y^{\prime} .
$$




\section{B. Microscanning}

Microscanning is the process of generating multiple images from a common scene by shifting either the scene or the image acquisition system. The shifting can be performed in a regular pattern or irregular pattern. Fig. 2 illustrates the microscanning process for a sequence of images $p_{k}, k=0 . . K-1$, with unchanging scene shifted between images, variable blur and noise, and fixed sampling grid. (Reverse shifting of the sampling grid for a fixed scene produces the same images.) Then, microscanned image $p_{k}$ is:

$$
p_{k}[m, n]=\iint s\left(x-x_{k}, y-y_{k}\right) h_{k}(m-x, n-y) d x d y+e_{k}[m, n],
$$

where $k$ is the index for the microscanning image, $\left(x_{k}, y_{k}\right)$ is the relative shift, $h_{k}$ is the acquisition PSF, and $e_{k}$ is the additive system noise.

Image acquisition (with blurring, sampling, and noise), digital processing (for registration, reconstruction, and restoration), and display of microscanned imaging is analyzed more easily in the Fourier frequency domain, regardless of whether digital image processing is performed in the spatial domain or the frequency domain. In the frequency domain, the Fourier transform of the microscanned image $p_{k}$ (the transform of Eq. 3) is:

$$
\hat{p}_{k}(u, v)=\sum_{\mu} \sum_{\nu} \hat{s}(u-\mu, v-\nu) \hat{h}_{k}(u-\mu, v-\nu) \exp ^{-i 2 \pi\left((u-\mu) x_{k}+(v-\nu) y_{k}\right)}+\hat{e}_{k}(u, v),
$$

where "^, indicates the Fourier transform. In Eq. 4, the frequency-domain equivalent for spatial-domain blurring by convolution in Eq. 3 is pointwise multiplication of the trans-

form coefficients of the scene and PSF. The frequency-domain equivalent for spatial-domain sampling is the double sum, which folds the transform coefficients.

The microscanned images must be registered relative to one another. In the frequency domain, the registered and combined microscanned images are:

$$
\begin{aligned}
\hat{p}(u, v)= & \frac{1}{K} \sum_{k=0}^{K-1} \hat{p}_{k}(u, v) \exp ^{i 2 \pi\left(u\left(x_{k}+\alpha_{k}\right)+v\left(y_{k}+\beta_{k}\right)\right)} \\
= & \frac{1}{K} \sum_{\mu} \sum_{\nu} \hat{s}(u-\mu, v-\nu) \sum_{k=0}^{K-1} \hat{h}_{k}(u-\mu, v-\nu) \exp ^{i 2 \pi\left(u \alpha_{k}+v \beta_{k}\right)} e x p^{i 2 \pi\left(\mu x_{k}+\nu y_{k}\right)} \\
& +\frac{1}{K} \sum_{k=0}^{K-1} \hat{e}_{k}(u, v) \exp ^{i 2 \pi\left(u\left(x_{k}+\alpha_{k}\right)+v\left(y_{k}+\beta_{k}\right)\right)}
\end{aligned}
$$

where $\left(\alpha_{k}, \beta_{k}\right)$ is the registration error for image $p_{k}$. Mathematically, the registered images are combined by addition. If registration is perfect, then $\alpha_{k}=\beta_{k}=0$ and each microscanned image is shifted to its proper position in the registered image.

In the Fourier frequency domain, reconstruction and restoration of the scene is the product of the Fourier transform of the registered image $\hat{p}$ by the filter transfer function $\hat{f}$. Then, the display process multiplies that result by the display transfer function $\hat{d}$ :

$$
\hat{r}(u, v)=\hat{p}(u, v) \hat{f}(u, v) \hat{d}(u, v) .
$$




\section{C. Fidelity analysis}

By Rayleigh's Theorem, the expected mean square error (MSE) of the CDC imaging system for an ensemble of scenes can be analyzed in either the spatial or frequency domain:

$$
\begin{aligned}
\epsilon^{2} & =\mathcal{E}\left\{\iint|r(x, y)-s(x, y)|^{2} d x d y\right\} \\
& =\mathcal{E}\left\{\iint|\hat{r}(u, v)-\hat{s}(u, v)|^{2} d u d v\right\} .
\end{aligned}
$$

The following analysis assumes that the power spectra of the scene ensemble and noise are known, that the scene and noise are uncorrelated, that co-aliased components of the sampled scene are uncorrelated, and that the noise between images is uncorrelated: ${ }^{24}$

$$
\begin{aligned}
\mathcal{E}\left\{\hat{s}(u, v) \hat{s}^{*}(u-\mu, v-\nu)\right\} & = \begin{cases}\hat{\Phi}_{s}(u, v) & \text { if }(\mu, \nu)=(0,0) \\
0 & \text { otherwise }\end{cases} \\
\mathcal{E}\left\{\hat{e}_{j}(u, v) \hat{e}_{k}^{*}(u, v)\right\} & = \begin{cases}\hat{\Phi}_{e_{k}}(u, v) & \text { if } j=k \\
0 & \text { otherwise }\end{cases} \\
\mathcal{E}\left\{\hat{s}(u-\mu, v-\nu) \hat{e}_{k}^{*}(u, v)\right\} & =0
\end{aligned}
$$

where the '*' superscript denotes complex conjugation, $\hat{\Phi}_{s}$ is the power spectra of the scene, and $\hat{\Phi}_{e_{k}}$ is the power spectra of the noise. For convenience, and without loss of generality, scenes are normalized so that the mean and variance are zero and one respectively.

The expected MSE $\epsilon^{2}$ for the CDC imaging system can be expressed in terms of the scene and noise power spectra, the acquisition transfer function, the relative shifts and registration errors, the reconstruction and restoration filter, and the display transfer function:

$$
\begin{aligned}
\epsilon^{2}= & \iint\left(\hat{\Phi}_{s}(u, v)-\hat{f}(u, v) \hat{d}(u, v) \hat{\Phi}_{s, p}^{*}(u, v)-\hat{f}^{*}(u, v) \hat{d}^{*}(u, v) \hat{\Phi}_{s, p}(u, v)\right. \\
& \left.+|\hat{f}(u, v)|^{2}|\hat{d}(u, v)|^{2} \hat{\Phi}_{p}(u, v)\right) d u d v
\end{aligned}
$$

where $\hat{\Phi}_{p}$ is the power spectrum of the registered image and $\hat{\Phi}_{s, p}$ is the cross-power spectrum of the scene and the registered image:

$$
\begin{aligned}
\hat{\Phi}_{p}(u, v)= & \mathcal{E}\left\{|\hat{p}(u, v)|^{2}\right\} \\
= & \frac{1}{K^{2}} \sum_{\mu} \sum_{\nu} \hat{\Phi}_{s}(u-\mu, v-\nu)\left|\sum_{k=0}^{K-1} \hat{h}_{k}(u-\mu, v-\nu) \mathcal{E}\left\{e x p^{i 2 \pi\left(u \alpha_{k}+v \beta_{k}\right)} e x p^{i 2 \pi\left(\mu x_{k}+\nu y_{k}\right)}\right\}\right|^{2} \\
& +\frac{1}{K^{2}} \sum_{k=0}^{K-1} \hat{\Phi}_{e_{k}}(u, v) \\
\hat{\Phi}_{s, p}(u, v)= & \mathcal{E}\left\{\hat{s}(u, v) \hat{p}^{*}(u, v)\right\} \\
= & \hat{\Phi}_{s}(u, v) \frac{1}{K} \sum_{k=0}^{K-1} \hat{h}_{k}^{*}(u, v) \mathcal{E}\left\{\exp ^{-i 2 \pi\left(u \alpha_{k}+v \beta_{k}\right)}\right\}
\end{aligned}
$$


If the distribution of the registration errors is known, the expressions for expected MSE can be analyzed with respect to those errors. For example, if $\alpha_{k}$ and $\beta_{k}$ are uniformly distributed on the intervals $\left[-\frac{1}{2 W_{x}}, \frac{1}{2 W_{x}}\right]$ and $\left[-\frac{1}{2 W_{y}}, \frac{1}{2 W_{y}}\right]$ respectively, then observing:

$$
\int_{-\frac{1}{2 W_{x}}}^{\frac{1}{2 W_{x}}} \int_{-\frac{1}{2 W_{y}}}^{\frac{1}{2 W_{y}}} \exp ^{i 2 \pi\left(u \alpha_{k}+v \beta_{k}\right)} W_{x} W_{y} d \alpha_{k} d \beta_{k}=\operatorname{sinc}\left(u / W_{x}\right) \operatorname{sinc}\left(v / W_{y}\right),
$$

the components of the expected MSE are:

$$
\begin{aligned}
\hat{\Phi}_{p}(u, v)= & \frac{1}{K^{2}} \operatorname{sinc}^{2}\left(u / W_{x}\right) \operatorname{sinc}^{2}\left(v / W_{y}\right) \\
& \sum_{\mu} \sum_{\nu} \hat{\Phi}_{s}(u-\mu, v-\nu)\left|\sum_{k=0}^{K-1} \hat{h}_{k}(u-\mu, v-\nu) \exp p^{i 2 \pi\left(\mu x_{k}+\nu y_{k}\right)}\right|^{2}+\frac{1}{K^{2}} \sum_{k=0}^{K-1} \hat{\Phi}_{e_{k}}(u, v) \\
\hat{\Phi}_{s, p}(u, v)= & \operatorname{sinc}\left(u / W_{x}\right) \operatorname{sinc}\left(v / W_{y}\right) \hat{\Phi}_{s}(u, v) \frac{1}{K} \sum_{k=0}^{K-1} \hat{h}_{k}^{*}(u, v) .
\end{aligned}
$$

$\hat{\Phi}_{p}$ is subject to the relative shifts $\left(x_{k}, y_{k}\right)$. For instance, if the number of microscanned images $K$ is $2, \hat{\Phi}_{p}$ for relative shifts $\left\{\left(x_{0}, y_{0}\right)=(0,0),\left(x_{1}, y_{1}\right)=(0,0.5)\right\}$ is the same as for relative shifts $\left\{\left(x_{0}, y_{0}\right)=(0.5,0),\left(x_{1}, y_{1}\right)=(0.5,0.5)\right\}$, but is different than for relative shifts $\left\{\left(x_{0}, y_{0}\right)=(0,0),\left(x_{1}, y_{1}\right)=(0.5,0)\right\}$ and for $\left\{\left(x_{0}, y_{0}\right)=(0,0.5),\left(x_{1}, y_{1}\right)=(0.5,0.5)\right\}$. Filters derived with respect to $\hat{\Phi}_{p}$ can vary depending on the relative shift pattern of microscanned images.

Fidelity $^{25}$ is a normalized measure of image quality based on the MSE:

$$
\mathcal{F}=1-\frac{\epsilon^{2}}{\iint \hat{\Phi}_{s}(u, v) d u d v} .
$$

The greatest fidelity possible is 1 , when the MSE is 0 . The next section derives superresolution reconstruction and restoration filters that maximize fidelity $\mathcal{F}$ (or equivalently minimize the MSE $\epsilon^{2}$ ).

\section{Super-Resolution Reconstruction and Restoration}

The techniques developed in this section are designed to perform reconstruction and restoration. The methods take as input multiple images which have been registered and produce a single output image with high fidelity and super-resolution.

\section{A. CDC Wiener filter}

For performance comparison, it is useful to derive the optimal CDC Wiener filter. ${ }^{24}$ Denoting MSE as a functional of the filter transfer function $\hat{f}(u, v)$ yields:

$$
\epsilon^{2}(\hat{f})=\iint L(u, v, \hat{f}) d u d v
$$


where

$$
\begin{aligned}
L(u, v, \hat{f})= & \hat{\Phi}_{s}(u, v)-\hat{f}(u, v) \hat{d}(u, v) \hat{\Phi}_{s, p}^{*}(u, v)-\hat{f}^{*}(u, v) \hat{d}^{*}(u, v) \hat{\Phi}_{s, p}(u, v) \\
& +|\hat{f}(u, v)|^{2}|\hat{d}(u, v)|^{2} \hat{\Phi}_{p}(u, v) .
\end{aligned}
$$

The optimal filter must satisfy:

$$
\frac{\partial L}{\partial \hat{f}}=\hat{f}^{*}(u, v)|\hat{d}(u, v)|^{2} \hat{\Phi}_{p}(u, v)-\hat{d}(u, v) \hat{\Phi}_{s, p}^{*}(u, v)=0,
$$

so the optimal filter is:

$$
\hat{f}_{w}(u, v)=\frac{\hat{\Phi}_{s, p}(u, v)}{\hat{\Phi}_{p}(u, v)} \frac{\hat{d}^{*}(u, v)}{|\hat{d}(u, v)|^{2}}=\frac{\hat{\Phi}_{s, \tilde{p}}(u, v)}{\hat{\Phi}_{\tilde{p}}(u, v)},
$$

where $\hat{\Phi}_{\tilde{p}}$ and $\hat{\Phi}_{s, \tilde{p}}$ are introduced to incorporate the effects of the display device on the image (and are used in the derivations of the small kernels in the following sections). The CDC Wiener filter cannot be implemented practically via spatial convolution because it is continuous and its support is the full extent of the image. As described in the next section, the computational costs of super-resolution reconstruction and restoration can be reduced by constraining the spatial support of the filter to a small kernel.

\section{B. Small-kernel Wiener filter}

The derivation of the small-kernel Wiener filter $f_{c}$ is conditioned on constraints imposed on its spatial support. The support of the kernel is a nonempty set of spatial discrete locations $C$, for which filter values can be nonzero. Except for locations in the support set, the filter value is 0 :

$$
f_{c}(x, y)=0, \quad \text { if }(x, y) \notin C .
$$

The larger the filter support, the better the performance, but small kernels can be highly effective. ${ }^{26}$

The optimal, spatially constrained filter is derived by minimizing the MSE $\epsilon^{2}$ with respect to the elements in $C$. Mathematically, it requires that:

$$
\frac{\partial \epsilon^{2}}{\partial f_{c}(x, y)}=0 \quad \forall(x, y) \in C .
$$

These constraints can be expressed in a system of linear equations: ${ }^{26}$

$$
\sum_{\left(x^{\prime}, y^{\prime}\right) \in C} \Phi_{\tilde{p}}\left(x-x^{\prime}, y-y^{\prime}\right) f_{c}\left(x^{\prime}, y^{\prime}\right)=\Phi_{s, \tilde{p}}(x, y), \quad \forall(x, y) \in C
$$

where $\Phi_{\tilde{p}}$ is the auto-correlation of the displayed image and $\Phi_{s, \tilde{p}}$ is the cross-correlation of the scene and the displayed image. The number of equations and the number of unknowns are both equal to the number of elements in the support set $C$, i.e., there are $|C|$ equations in $|C|$ unknowns. 


\section{C. Parametric cubic convolution}

Piecewise cubic convolution is a popular interpolation method for image reconstruction that is traditionally implemented by separable convolution with a small one-dimensional kernel consisting of piecewise cubic polynomials. ${ }^{27,28}$ This popular method can be generalized to two dimensions and can be reformulated by relaxing constraints to perform reconstruction and restoration in one-pass with small-kernel convolution. ${ }^{29}$ With constraints for symmetry, continuity, and smoothness, the two-dimensional kernel with support $[-2,2] \times[-2,2]$ has five parameters $\left\{a_{1}, a_{2}, a_{3}, a_{4}, a_{5}\right\}$ :

$$
f_{p}(x, y)=f_{0}(x, y)+a_{1} f_{1}(x, y)+a_{2} f_{2}(x, y)+a_{3} f_{3}(x, y)+a_{4} f_{4}(x, y)+a_{5} f_{5}(x, y),
$$

where $f_{0}-f_{5}$ are defined in Fig. 3. This kernel, designated 2D-5PCC-R (to distinguish it from two-dimensional piecewise cubic interpolation ${ }^{30}$ ), is a continuous function.

The optimal 2D-5PCC-R kernel $f_{p}$ for an ensemble of scenes can be derived by minimizing the MSE $\epsilon^{2}$ with respect to the five parameters. Computing the partial derivatives of $\epsilon^{2}$ with respect to the parameters, and solving for simultaneous equality with zero:

$$
\frac{\partial \epsilon^{2}}{\partial a_{1}}=\frac{\partial \epsilon^{2}}{\partial a_{2}}=\frac{\partial \epsilon^{2}}{\partial a_{3}}=\frac{\partial \epsilon^{2}}{\partial a_{4}}=\frac{\partial \epsilon^{2}}{\partial a_{5}}=0
$$

yields five equations for the optimal parameter value:

$$
\begin{aligned}
& \iint \hat{f}_{i}(u, v)\left(\operatorname{Re}\left\{\hat{\Phi}_{s, \tilde{p}}(u, v)\right\}-\hat{f}_{0}(u, v) \hat{\Phi}_{\tilde{p}}(u, v)\right) d u d v \\
& \quad=\iint \hat{f}_{i}(u, v)\left(\hat{f}_{p}(u, v)-\hat{f}_{0}(u, v)\right) \hat{\Phi}_{\tilde{p}}(u, v) d u d v, \quad i=1 . .5 .
\end{aligned}
$$

\section{Experimental results}

This section presents experimental results for a simulated imaging system and for real images. In the simulation, the scene is a high resolution digital image. The simulated scene is blurred, sampled, and degraded by noise (by digital processing) to produce simulated microscanned images. These images are reconstructed and restored by the optimal CDC Wiener filter, the small-kernel Wiener filter, 2D-5PCC-R, Shift-and-Add (with Wiener deconvolution, denoted $\mathrm{SA}+$ Wiener), ${ }^{20,31}$ and Norm 2 Data with $L_{1}$ Regularization (denoted Norm 2 Data). ${ }^{23,31}$

The super-resolution computations for the optimal Wiener filter are implemented in the Fourier frequency domain by multiplying the filter defined in Eq. 17 by the Fourier transform of the registered, combined images defined in Eq. 5. The computations for the small-kernel Wiener filter and 2D-5PCC-R are implemented in the spatial domain, for computational efficiency, by convolving the registered combined images with the small kernels defined by the solutions for Eq. 20 and Eq. 23, respectively. Both SA+Wiener and Norm 2 Data were developed at the Multi-Dimensional Signal Processing (MDSP) research lab at the University 
of California at Santa Cruz. ${ }^{31}$ Norm 2 Data is an iterative super-resolution method and these experiments use default parameter values (except the deconvolution kernel) of the software.

For simulated imaging, all resulting images are compared to the original scene. Because the phase-shifts between the simulated scene and the microscanned images are known, the simulation allows true quantitative measures of reconstruction and restoration performance. The super-resolution methods also are applied to real images acquired by panning an infrared camera slowly across a fixed scene. Because the true scene values are unknown, quantitative evaluation is not possible. Also, the microscanning shifts must be estimated, so the results are impacted by registration error. Nonetheless, real images are useful for qualitatively demonstrating the effectiveness of the small-kernel methods in practice.

\section{A. Simulation results}

Fig. 4(A) illustrates a $256 \times 256$ digital image acquired by aerial photography ${ }^{32}$ that is used as a simulated scene and it is therefore the ideal super-resolution image. The simulated scene is blurred by a Gaussian low-pass filter to simulate acquisition blurring:

$$
\hat{h}(u, v)=\exp ^{-\left(u^{2}+v^{2}\right)}
$$

so the system transfer function at the Nyquist limit along each axis is $\hat{h}(0.0,0.5)=\hat{h}(0.5,0.0)$ $=0.779$. After blurring, sixteen $64 \times 64$ low-resolution images are created with simulated microscanning at quarter-pixel intervals along each axis. In each simulated microscanned image, Gaussian white noise is added to each pixel so that the blurred-signal-to-noise ratio $(\mathrm{BSNR})$ is $30 d B$ :

$$
\mathrm{BSNR}=10 \log _{10}\left(\sigma_{p}^{2} / \sigma_{e}^{2}\right)
$$

where $\sigma_{p}^{2}$ is the variance of the blurred microscanned images (after blurring and before additive noise) and $\sigma_{e}^{2}$ is the variance of the noise. Fig. 4(B) illustrates one of the microscanned images (the reference image $p_{0}$ ) interpolated back to $256 \times 256$ resolution by nearest-neighbor interpolation to show the granularity of the sampling. Fig. 4(C) illustrates a higher quality interpolation using cubic o-Moms. ${ }^{33}$ Four of the sixteen microscanned images are used for deriving the reconstruction and restoration filters and for super-resolution processing:

\begin{tabular}{|c|c|c|c|}
\hline$\times$ & $\mathrm{O}$ & $\times$ & $\mathrm{O}$ \\
\hline $\mathrm{O}$ & $\mathrm{O}$ & $\mathrm{O}$ & $\mathrm{O}$ \\
\hline$\times$ & $\mathrm{O}$ & $\times$ & $\mathrm{O}$ \\
\hline $\mathrm{O}$ & $\mathrm{O}$ & $\mathrm{O}$ & $\mathrm{O}$ \\
\hline
\end{tabular}

where ' $x$ ' and ' $O$ ' respectively stand for locations (at quarter-pixel intervals) with and without samples. 
The actual scene power spectrum is used to derive the optimal CDC Wiener filter in order to benchmark the optimal fidelity. The power spectrum for optimizing the small-kernel Wiener filter and the 2D-5PCC-R filter, however, is estimated (as typically is required in practice) using the power spectrum model of a two-dimensional isotropic Markov random field (MRF). ${ }^{34}$ The model can be fitted to the image power spectrum ${ }^{30}$ or interactively parameterized for visual quality (as done here). The MRF autocorrelation is:

$$
\Phi_{s}(x, y)=\exp ^{-\sqrt{x^{2}+y^{2}} / \rho},
$$

where $\rho$ is the mean spatial detail (MSD) of the scene in pixel units. MSD can be interpreted as the average size of the details in the scene. In terms of the Hankel transform, ${ }^{35}$ the power spectrum of the isotropic MRF is:

$$
\hat{\Phi}_{s}(u, v)=\frac{2 \pi \rho^{2}}{\left(1+4 \pi^{2} \rho^{2}\left(u^{2}+v^{2}\right)\right)^{\frac{3}{2}}} .
$$

The CDC Wiener filter, small-kernel Wiener filter with support limited to $[-2,2] \times[-2,2]$, and 2D-5PCC-R filter were derived for this simulation based on the isotropic MRF scene model with $M S D=4$ pixels. Fig. 5 illustrates the small-kernel Wiener PSF (in Fig. 5(A)) and the 2D-5PCC-R PSF (in Fig. 5(B)). The optimal parameter values for 2D-5PCC-R are $a_{1}=74.176, a_{2}=-95.360, a_{3}=16.804, a_{4}=-0.967$, and $a_{5}=0.238$.

Fig. 4(D)-(H) presents the simulation results for the super-resolution methods. To limit boundary effects, the borders of all resulting images are cleared. Visually, the super-resolution images produced by all of the restoration and reconstruction filters (in Fig. 4(D)-(H)) are better than provided by a single frame (in Fig. 4(B)-(C)). For example, the small, lightly colored rectangle in the upper-left quadrant between the diagonal runway and the left-most vertical runway are clearer in the super-resolution images. The images produced by the five super-resolution methods are of similar visual quality, but the CDC Wiener filter appears to produce the best image and $\mathrm{SA}+$ Wiener appears to produce the worst image. The image produced by the small-kernel Wiener filter appears to be slightly sharper than the image produced by 2D-5PCC-R.

Table 1 lists the quantitative fidelity and computational costs for the various methods with the simulation. The computational costs were measured in seconds, averaged over multiple runs with MATLAB 6.5 Release 13 on a IBM R32 (Intel Pentium M 1.8GHz CPU, 256MB RAM, MS Windows XP Professional 2002). The optimal CDC Wiener filter (which uses the actual scene power spectrum) has the highest fidelity, as expected mathematically. The CDC Wiener filter requires preprocessing to compute the filter (which then can be used to filter multiple images) and requires forward and inverse Fourier transforms to apply the filter. As expected, the image from cubic o-Moms with a single frame has the lowest fidelity. The iterative method, Norm 2 Data, has fidelity nearly equal to the CDC Wiener filter, but 
requires 50 iterations and nearly 50 seconds for Fig. 4(H). Of the three small-kernel filters which can be efficiently applied by spatial convolution, the small-kernel Wiener filter has somewhat higher fidelity than 2D-5PCC-R and SA+Wiener.

The visual and quantitative results for the simulation indicate that the small-kernel Wiener filter and 2D-5PCC-R effectively improve image quality with efficient spatial-domain processing.

\begin{tabular}{|l|c|c|c|c|c|c|}
\hline & CDC Wiener & Small-kernel Wiener & 2D-5PCC-R & O-Moms & SA+Wiener & Norm 2 Data \\
\hline Fidelity & 0.980 & 0.975 & 0.966 & 0.928 & 0.966 & 0.979 \\
\hline $\begin{array}{c}\text { Preprocessing } \\
\text { time (sec) }\end{array}$ & 0.521 & 0.781 & 0.940 & 0.000 & 0.150 & 0.000 \\
\hline $\begin{array}{c}\text { Filtering } \\
\text { time (sec) }\end{array}$ & 0.121 & 0.030 & 0.030 & 0.120 & 0.380 & 49.121 \\
\hline
\end{tabular}

Table 1. Fidelity and computational costs of various methods.

\section{B. Results for real images}

The super-resolution reconstruction and restoration methods require characterizations of the system, including the noise power spectrum and acquisition transfer function. Noise can be characterized accurately from flat-field calibration images or from regions of uniform background of acquired images. The transfer function can be estimated to frequencies beyond the Nyquist limit using a knife-edge technique with images for various sample-scene phase shifts. ${ }^{36}$ Microscanning provides such images.

For this experiment with real images, the acquisition transfer function of an infrared camera system was estimated from microscanned images of a four-bar target. The camera platform was microscanned as low-resolution images were recorded. Fig. 6 illustrates a small piece of one of the $256 \times 256$ low-resolution images from the microscanned sequence. Employing the super-resolution knife-edge technique, ${ }^{36}$ a sequence of 120 low-resolution images of the bar target was registered with the subpixel accuracy (to 0.25 pixel) and averaged. Fig. 7 illustrates the resulting super-resolution horizontal slice across the four-bar targets, superimposed on the model of the bar target scene estimated by a thresholding the registered slice. Fig. 8 illustrates the modulation transfer function (MTF) estimated by the CDC Wiener filter. For this experiment, the two-dimensional acquisition transfer function was modeled as the separable product of the one-dimensional estimate:

$$
\hat{h}(u, v)=\hat{h}_{x}(u) \hat{h}_{x}(v)
$$


A more accurate estimate of the two-dimensional acquisition transfer function could be made from two or more slices.

In this experiment, the goal of super-resolution reconstruction and restoration is to generate a $1024 \times 1024$, high-fidelity image from multiple microscanned $256 \times 256$ images. Fig. 9(A) illustrates one of a microscanned sequence of low-resolution images from the infrared camera system, interpolated to $1024 \times 1024$ by nearest neighbor interpolation. The scene is modeled as a MRF with $M S D$ equal to 8 pixels. The BSNR was estimated to be $30 d B$.

Fig. 10 illustrates the small-kernel Wiener and cubic convolution reconstruction and restoration kernels, based on these system characterizations. The optimal parameter values for 2D-5PCC-R are $a_{1}=-1.052, a_{2}=-7.033, a_{3}=7.584, a_{4}=-1.123$, and $a_{5}=0.137$. The small-kernel Wiener PSF provides more sharpening.

Fig. 9(B) illustrates a $1024 \times 1024$ image reconstructed from a single image by cubic oMoms. Fig. 9(C)-(F) illustrate $1024 \times 1024$ images reconstructed and restored with the smallkernel Wiener filter, 2D-5PCC-R, SA+Wiener, and Norm 2 Data. These results are generated from just three $256 \times 256$, microscanned low-resolution images with different relative shifts. The relative shift pattern for the three images is:

\begin{tabular}{|c|c|c|c|}
\hline$\times$ & $\times$ & $\mathrm{O}$ & $\times$ \\
\hline $\mathrm{O}$ & $\mathrm{O}$ & $\mathrm{O}$ & $\mathrm{O}$ \\
\hline $\mathrm{O}$ & $\mathrm{O}$ & $\mathrm{O}$ & $\mathrm{O}$ \\
\hline $\mathrm{O}$ & $\mathrm{O}$ & $\mathrm{O}$ & $\mathrm{O}$ \\
\hline
\end{tabular}

To limit boundary effects, the borders of the processed images are cleared. Visually, resulting images in Fig. 9(C), (D) and (F) from super-resolution reconstruction and restoration are substantially better than a single image in either Fig. 9(A)-(B). Fig. 9(E) from the $\mathrm{SA}+$ Wiener super-resolution method is blurred. The super-resolution image from the smallkernel Wiener filter is slightly sharper than the image from 2D-5PCC-R, consistent with the simulation results.

\section{Conclusion}

Images with super-resolution and high fidelity are required in many imaging applications. This paper investigates super-resolution methods for microscanning imaging systems that efficiently implement reconstruction and restoration with small convolution kernels. The approach is based on an end-to-end system model that accounts for the fundamental tradeoff in imaging system design between acquisition and aliasing. The system model also accounts for noise, mis-registration of the low-resolution images, and the display system. Computationally efficient reconstruction and restoration is realized by using one-pass convolution with small kernels. 
This paper develops two small convolution kernels for improved resolution and fidelity: the spatially constrained Wiener filter and a parametric cubic convolution (designated 2D5PCC-R). Subject to constraints, both are optimized with respect to maximum end-to-end system fidelity. Experimental results for a simulated imaging system and for real images indicate the effectiveness of the small-kernel methods for increasing resolution and fidelity. Visually, the super-resolution images from the small-kernel Wiener filter are slightly sharper than images from 2D-5PCC-R, but both small-kernel methods yield significant quantitative and qualitative improvements.

Additional work is required to develop efficient implementations of the small-kernel superresolution methods. Even with the efficiency of one-pass restoration and reconstruction using small kernels, super-resolution processing requires significant processing. Each lowresolution image pixel in the kernel's region-of-support around each super-resolution pixel contributes to the output value. Super-resolution processing of $K$ images, with kernel support of $[-S, S] \times[-S, S]$ pixels and super-resolution increase of $R \times R$, requires $4 K S^{2} R^{2}$ floating-point multiplications and additions (MADDs). If the distribution of low-resolution

pixels varies with respect to the super-resolution pixels, multiple kernels (each with $K S^{2}$ weights) should be used in the computation. These issues make implementation, especially in hardware, challenging.

\section{References}

1. M. G. Kang and S. Chaudhuri, "Super-resolution image reconstruction," IEEE Signal Processing Magazine 20(3), 19-20 (2003).

2. E. Choi, J. Choi, and M. G. Kang, "Super-resolution approach to overcome physical limitations of imaging sensors: An overview," International Journal of Imaging Systems and Technology 14(2), 36-46 (2004).

3. S. C. Park, M. K. Park, and M. G. Kang, "Super-Resolution Image Reconstruction: A Technical Overview," IEEE Signal Processing Magazine 20(3), 21-36 (2003).

4. R. C. Hardie, K. J. Barnard, J. G. Bognar, E. E. Armstrong, and E. A. Watson, "Highresolution image reconstruction from a sequence of rotated and translated frames and its application to an infrared imaging system," Optical Engineering 37(1), 247-260 (1998).

5. M. Elad and A. Feuer, "Restoration of a Single Superresolution Image from Several Blurred, Noisy, and Undersampled Measured Images," IEEE Transactions on Image Processing 6(12), 1646-1658 (1997).

6. C. L. L. Hendriks and L. J. V. Vliet, "Improving Resolution to Reduce Aliasing in an Undersampled Image Sequence," SPIE 3965, 1-9 (2000).

7. H. Foroosh, J. B. Zerubia, and B. Marc, "Extension of Phase Correlation to Subpixel Registration," IEEE Transactions on Image Processing 11(3), 188-200 (2002). 
8. M. Irani and S. Peleg, "Improving Resolution by Image Registration," CVGIP: Graphical Models and Image Processing 53(3), 231-239 (1991).

9. M. S. Alam, J. G. Bognar, R. C. Hardie, and B. J. Yasuda, "Infrared Image Registration and High-Resolution Reconstruction Using Multiple Translationally Shifted Aliased Video Frames," IEEE Transactions on Instrumentation and Measurement 49(5), 915-923 (2000).

10. T. M. Lehmann, C. Gonner, and K. Spitzer, "Survey: Interpolation Methods in Medical Image Processing," IEEE Transactions on Medical Imaging 18(11), 1049-1075 (1999).

11. M. L. Stein, Interpolation of Spatial Data: Some Theory for Kriging (Springer-Verlag, New York, NY, 1999).

12. J. Ruiz-Alzola, C. Alberola-López, and C. F. Westin, "Adaptive Kriging Filters for Multidimensional Signal Processing," Signal Processing 85(2), 413-439 (2005).

13. A. K. Katsaggelos, Digital Image Restoration (Springer-Verlag, New York, NY, 1991).

14. R. L. Lagendijk and J. Biemond, "Basic Methods for Image Restoration and Identification," in Handbook of Image and Video Processing (A. Bovik, Ed. Academic Press,San Diego CA, 2000).

15. M. K. Ng and N. K. Bose, "Mathematical analysis of super-resolution methodology," IEEE Signal Processing Magazine 20(3), 62-74 (2003).

16. R. Y. Tsai and T. S. Huang, "Multiframe Image Restoration and Registration," in $A d$ vances in Computer Vision and Image Processing, pp. 317-339 (Greenwich, CT, 1984).

17. S. P. Kim, N. K. Bose, and H. M. Valenzuela, "Recursive Reconstruction of High Resolution Image from Noisy Undersampled Multiframes," IEEE Transactions on Acoustics, Speech, and Signal Processing 38(6), 1013-1027 (1990).

18. S. P. Kim and W.-y. Su, "Recursive High-Resolution Reconstruction of Blurred Multiframe Images," IEEE Transactions on Image Processing 2(4), 534-539 (1993).

19. R. R. Schultz and R. L. Stevenson, "Extraction of High-Resolution Frames from Video Sequences," IEEE Transactions on Image Processing 5(6), 996-1011 (1996).

20. M. Elad and Y. Hel-Or, "A Fast Super-Resolution Reconstruction Algorithm for Pure Translational Motion and Common Space-Invariant Blur," IEEE Transactions on Image Processing 10(8), 1187-1193 (2001).

21. N. Nguyen and P. Milanfar, "A computationally efficient super-resolution image reconstruction algorithm," IEEE Transactions on Image Processing 10(4), 573-583 (2001).

22. S. Farsiu, D. Robinson, M. Elad, and P. Milanfar, "Advances and challenges in superresolution," International Journal of Imaging Systems and Technology 14(2), 47-57 (2004).

23. S. Farsiu, D. Robinson, M. Elad, and P. Milanfar, "Fast and robust multiframe super resolution," IEEE Transactions on Image Processing 13(10), 1327-1344 (2004). 
24. C. L. Fales, F. O. Huck, J. A. McCormick, and S. K. Park, "Wiener Restoration of Sampled Image Data: End-to-End Analysis," Journal of the Optical Society of America A 5(3), 300-314 (1988).

25. E. H. Linfoot, "Transmission Factors and Optical Design," Journal of the Optical Society of America 46(9), 740-752 (1956).

26. S. E. Reichenbach and S. K. Park, "Small Convolution Kernels for High-Fidelity Image Restoration," IEEE Transactions on Signal Processing 39(10), 2263-2274 (1991).

27. R. G. Keys, "Cubic Convolution Interpolation for Digital Image Processing," IEEE Transactions on Acoustics, Speech, and Signal Processing 29(6), 1153-1160 (1981).

28. S. K. Park and R. A. Schowengerdt, "Image Reconstruction by Parametric Cubic Convolution," Computer Vision, Graphics, and Image Processing 23, 258-272 (1983).

29. S. E. Reichenbach and J. Shi, "Two-dimensional Cubic Convolution for One-pass Image Restoration and Reconstruction," in International Geoscience and Remote Sensing Symposium, pp. 2074-2076 (IEEE, 2004).

30. J. Shi and S. E. Reichenbach, "Image Image Interpolation by Two-Dimensional Parametric Cubic Convolution," IEEE Transactions on Image Processing, to appear.

31. P. Milanfar, "Resolution Enhancement Software. www.soe.ucsc.edu/ milanfar/SRSoftware.htm (2004)".

32. Nebraska Department of Natural Resources, "Digital Orthophoto Quadrangle Database. www.dnr.state.ne.us/databank/coq.html (2004)".

33. T. Blu, P. Thévenaz, and M. Unser, "MOMS: maximal-order interpolation of minimal support," IEEE Transactions on Image Processing 10(7), 1069-1080 (2001).

34. R. A. Schowengerdt, Remote Sensing: Models and Methods for Image Processing, 2nd ed. (Academic Press, Orlando, FL, 1997).

35. K. R. Castleman, Digital Image Processing (Prentice-Hall, Englewood Cliffs, NJ, 1979).

36. S. E. Reichenbach, S. K. Park, and R. Narayanswamy, "Characterizing Digital Image Acquisition Devices," Optical Engineering 30(2), 170-177 (1991). 


\section{List of Figure Captions}

Fig. 1 End-to-end model of the digital imaging process.

Fig. 2 Microscanning produces multiple images.

Fig. 3 Components for the 2D-5PCC-R cubic convolution kernel for super-resolution restoration and reconstruction in Eq. 21.

Fig. 4 Simulation results.

Fig. 5 The small reconstruction and restoration kernels for the simulation experiment.

Fig. 6 A low-resolution infrared image of a four-bar target used for estimating the acquisition transfer function.

Fig. 7 Estimated acquisition transfer function $\hat{h}_{x}(u)$.

Fig. 8 Estimated acquisition transfer function $\hat{h}_{x}(u)$.

Fig. 9 Super-resolution results for a microscanned infrared system.

Fig. 10 The small reconstruction and restoration kernels for the real image experiment. 


\section{Continuous}

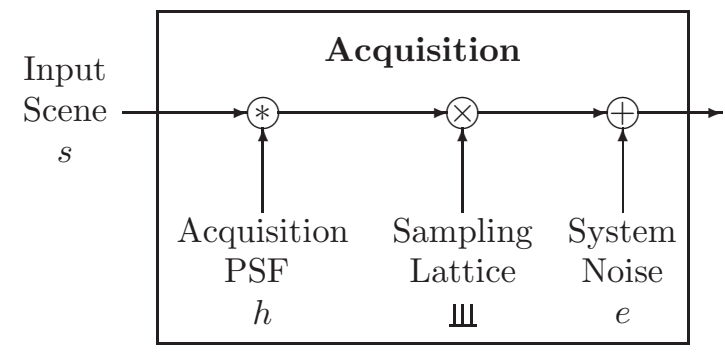

Discrete

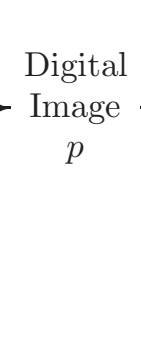

Continuous

\begin{tabular}{|cc|}
$\begin{array}{c}\text { Restoration } \\
\text { Reconstruction }\end{array}$ & Display \\
Filter & Display \\
PSF & PSF \\
$f$ & $d$ \\
\hline
\end{tabular}

Fig. 1. End-to-end model of the digital imaging process.

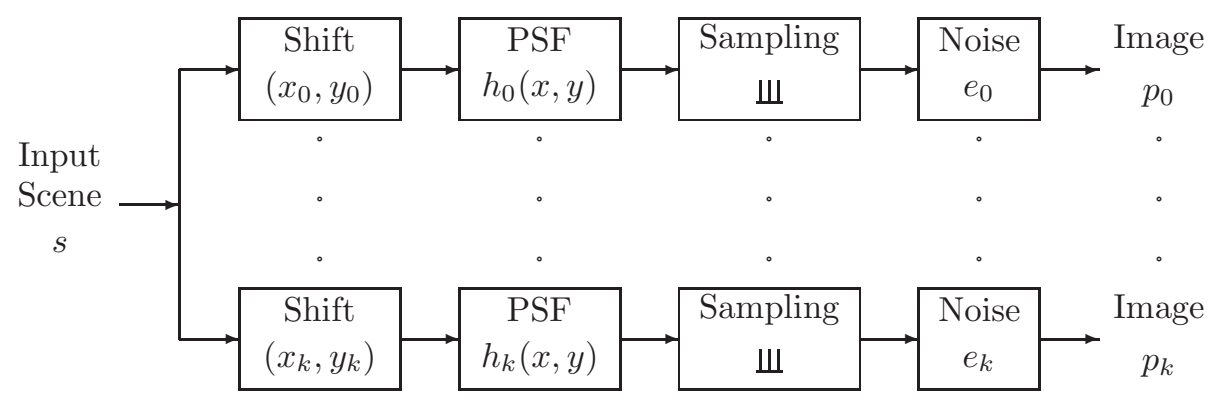

Fig. 2. Microscanning produces multiple images. 


$$
\begin{aligned}
& f_{0}(x, y)=\left\{\begin{array}{l}
x^{2} y^{2}-x^{2}-y^{2}+1 \\
\left(2 x y^{2}-2 x-2 y^{2}+2\right)(x-2)^{2} \\
(4 x y-4 y-4 x+4)(x-2)^{2}(y-2)^{2} \\
\left(2 x^{2} y-2 y-2 x^{2}+2\right)(y-2)^{2}
\end{array}\right. \\
& 0 \leq x \leq 1,0 \leq y \leq 1 \\
& 1<x \leq 2,0 \leq y \leq 1 \\
& 1<x \leq 2,1<y \leq 2 \\
& 0 \leq x \leq 1,1<y \leq 2 \\
& f_{1}(x, y)=\left\{\begin{array}{l}
x^{3} y^{3}-x^{2} y^{2} \\
\left(5 x y^{3}-4 x y^{2}-4 y^{3}+3 y^{2}\right)(x-2)^{2} \\
(9 x y-8 y-8 x+7)(x-2)^{2}(y-2)^{2} \\
\left(5 x^{3} y-4 x^{2} y-4 x^{3}+3 x^{2}\right)(y-2)^{2}
\end{array}\right. \\
& 0 \leq x \leq 1,0 \leq y \leq 1 \\
& 1<x \leq 2,0 \leq y \leq 1 \\
& 1<x \leq 2,1<y \leq 2 \\
& 0 \leq x \leq 1,1<y \leq 2 \\
& f_{2}(x, y)=\left\{\begin{array}{l}
x^{3} y^{2}-2 x^{2} y^{2}+x^{2} y^{3} \\
\left(4 x y^{3}-3 x y^{2}-3 y^{3}+2 y^{2}\right)(x-2)^{2} \\
(8 x y-7 y-7 x+6)(x-2)^{2}(y-2)^{2} \\
\left(4 x^{3} y-3 x^{2} y-3 x^{3}+2 x^{2}\right)(y-2)^{2}
\end{array}\right. \\
& 0 \leq x \leq 1,0 \leq y \leq 1 \\
& 1<x \leq 2,0 \leq y \leq 1 \\
& 1<x \leq 2,1<y \leq 2 \\
& 0 \leq x \leq 1,1<y \leq 2 \\
& f_{3}(x, y)=\left\{\begin{array}{l}
x^{3}-x^{2}-y^{2}+y^{3} \\
\left(2 x y^{3}-2 x y^{2}+x+y^{2}-y^{3}-1\right)(x-2)^{2} \\
(4 x y-3 x-3 y+2)(x-2)^{2}(y-2)^{2} \\
\left(2 x^{3} y-2 x^{2} y+y+x^{2}-x^{3}-1\right)(y-2)^{2}
\end{array}\right. \\
& 0 \leq x \leq 1,0 \leq y \leq 1 \\
& 1<x \leq 2,0 \leq y \leq 1 \\
& 1<x \leq 2,1<y \leq 2 \\
& 0 \leq x \leq 1,1<y \leq 2 \\
& f_{4}(x, y)=\left\{\begin{array}{l}
5 x^{2}-6 x^{2} y^{2}+5 y^{2}-4 \\
\left(13 y^{2}-14 x y^{2}+12 x-11\right)(x-2)^{2} \\
(30 x+30 y-32 x y-28)(x-2)^{2}(y-2)^{2} \\
\left(13 x^{2}-14 x^{2} y+12 y-11\right)(y-2)^{2}
\end{array}\right. \\
& 0 \leq x \leq 1,0 \leq y \leq 1 \\
& 1<x \leq 2,0 \leq y \leq 1 \\
& 1<x \leq 2,1<y \leq 2 \\
& 0 \leq x \leq 1,1<y \leq 2 \\
& f_{5}(x, y)=\left\{\begin{array}{l}
4 x^{2}-3 x^{2} y^{2}+4 y^{2}-4 \\
\left(5 y^{2}-4 x y^{2}+8 x-8\right)(x-2)^{2} \\
(4 x+4 y-7)(x-2)^{2}(y-2)^{2} \\
\left(5 x^{2}-4 x^{2} y+8 y-8\right)(y-2)^{2}
\end{array}\right. \\
& 0 \leq x \leq 1,0 \leq y \leq 1 \\
& 1<x \leq 2,0 \leq y \leq 1 \\
& 1<x \leq 2,1<y \leq 2 \\
& 0 \leq x \leq 1,1<y \leq 2
\end{aligned}
$$

Fig. 3. Components for the 2D-5PCC-R cubic convolution kernel for superresolution restoration and reconstruction in Eq. 21. 


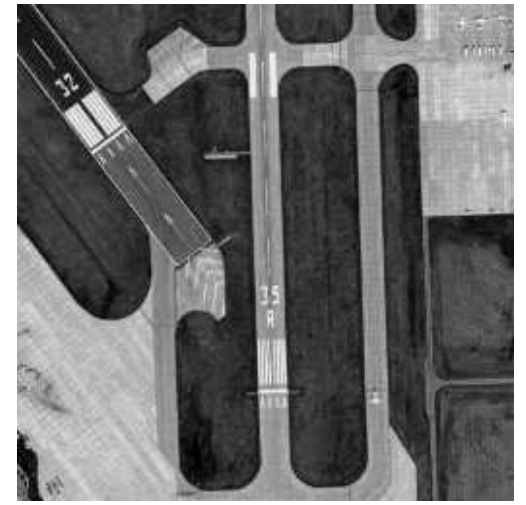

A. $256 \times 256$ simulated scene

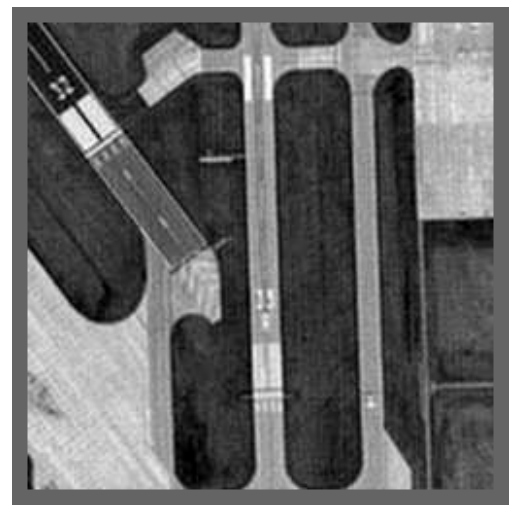

D. CDC Wiener

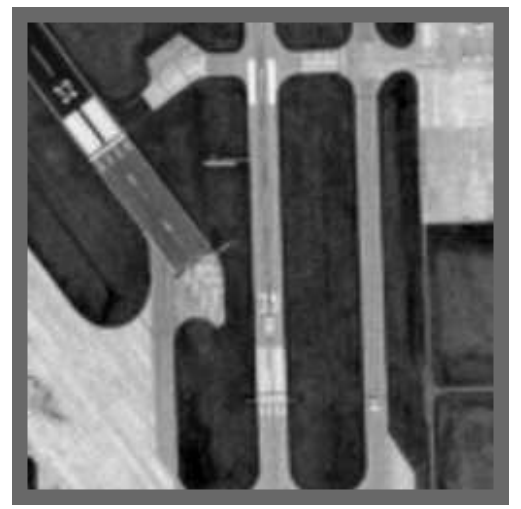

G. SA+Wiener

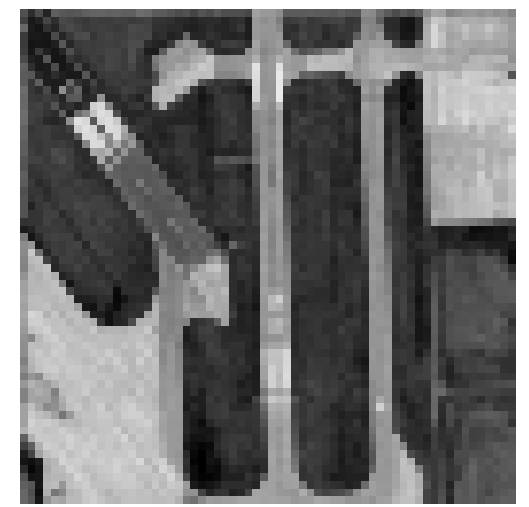

B. Microscanned image

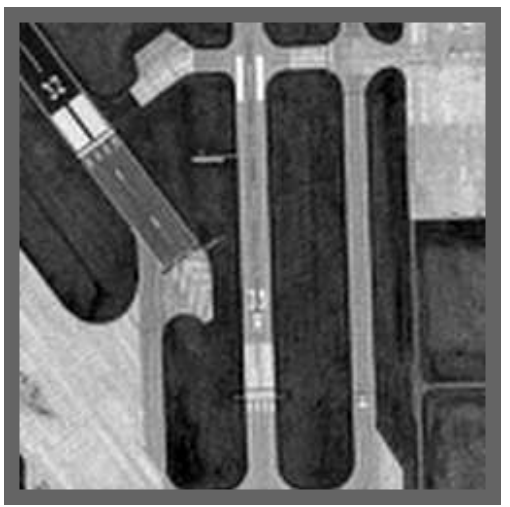

E. Small-kernel Wiener

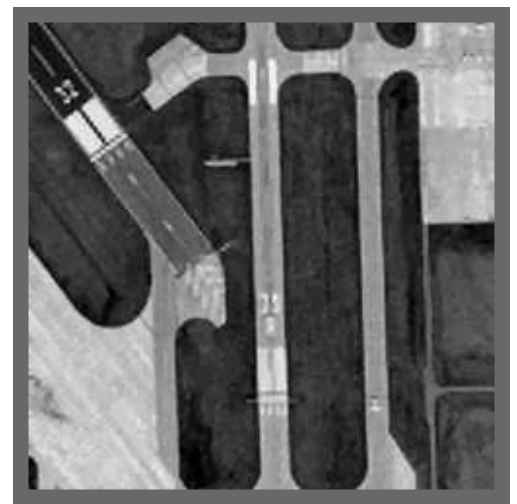

H. Norm 2 Data

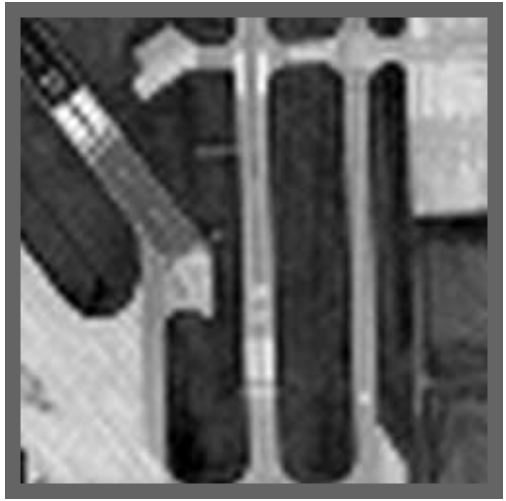

C. Cubic o-Moms

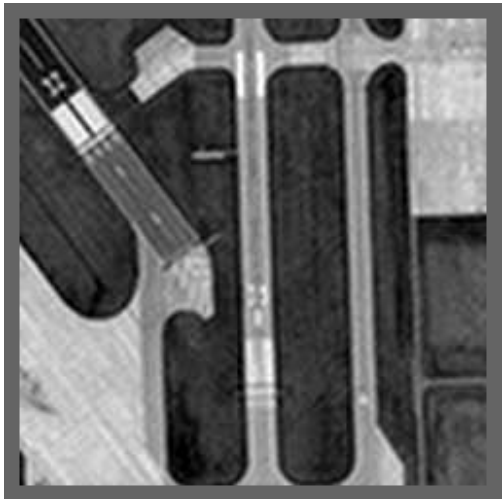

F. 2D-5PCC-R

Fig. 4. Simulation results: $(A)$ the $256 \times 256$ simulated scene and ideal superresolution image; (B) a single, $64 \times 64$, microscanned image reconstructed to $256 \times 256$ with nearest-neighbor interpolation; (C) a single microscanned image reconstructed to $256 \times 256$ with cubic o-Moms interpolation; (D)-(H) super-resolution image restored and reconstructed to $256 \times 256$ from four microscanned images by the CDC Wiener filter, small-kernel Wiener filter, 2D5PCC-R, SA+Wiener, and Norm 2 Data. 


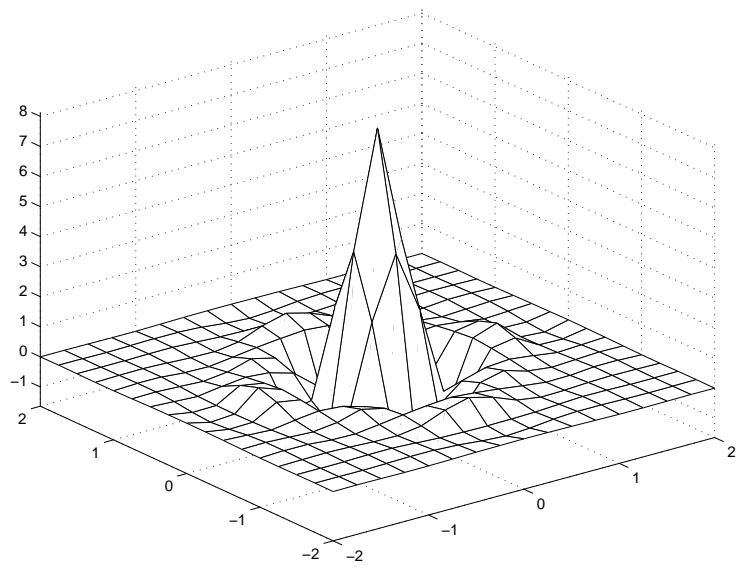

A. Small-kernel Wiener filter

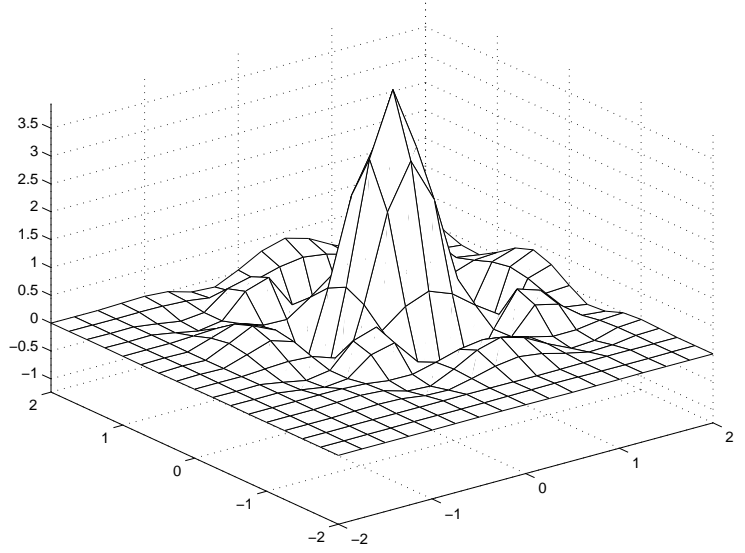

B. 2D-5PCC-R

Fig. 5. The small reconstruction and restoration kernels for the simulation experiment.

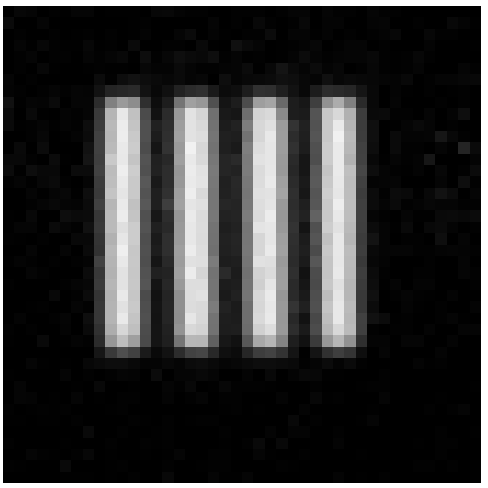

Fig. 6. A low-resolution infrared image of a four-bar target used for estimating the acquisition transfer function. 


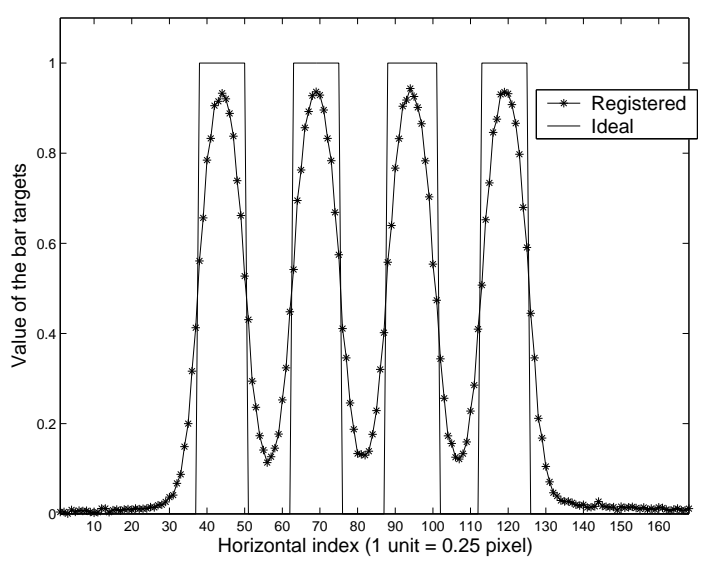

Fig. 7. Super-resolution average scan of the bar target.

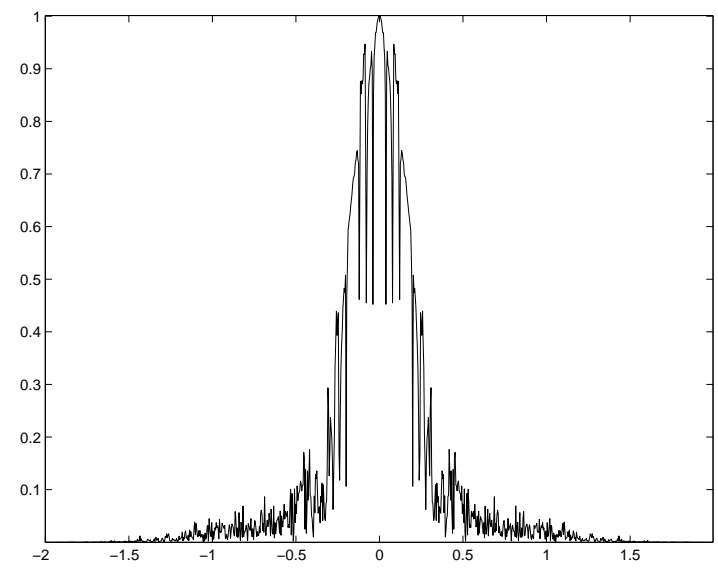

Fig. 8. Estimated acquisition transfer function $\hat{h}_{x}(u)$. 


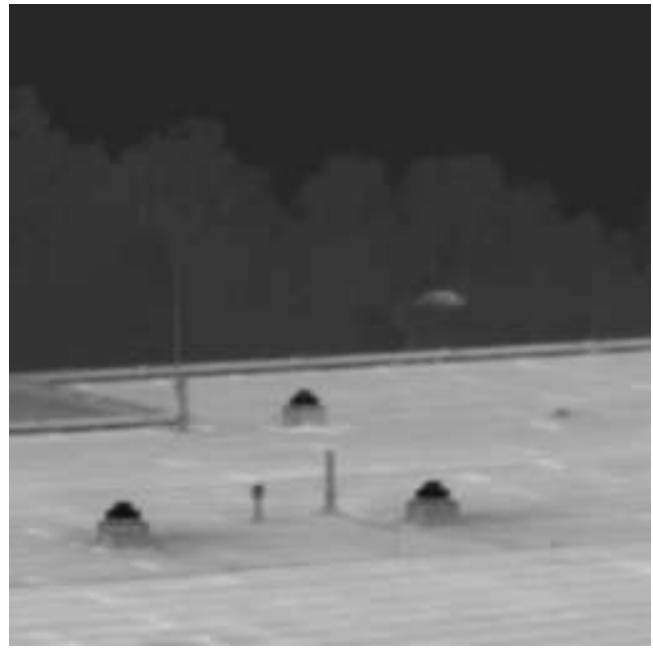

A. $256 \times 256$ frame

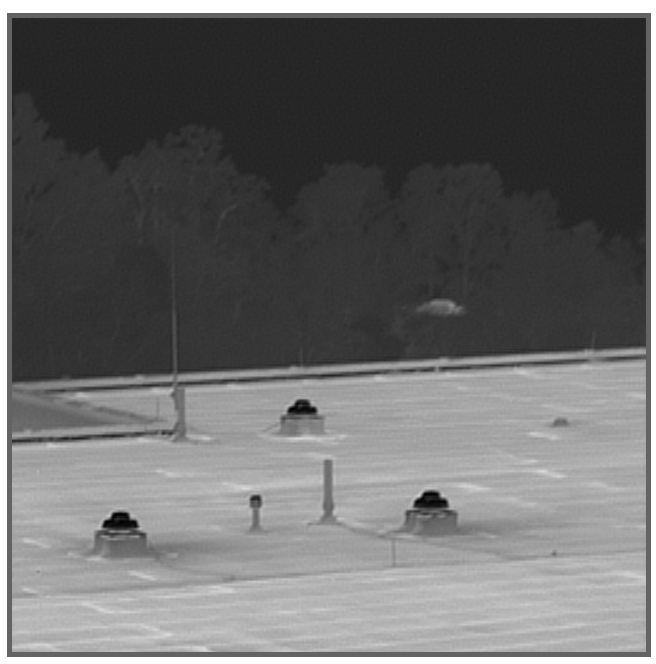

C. Small-kernel Wiener filter

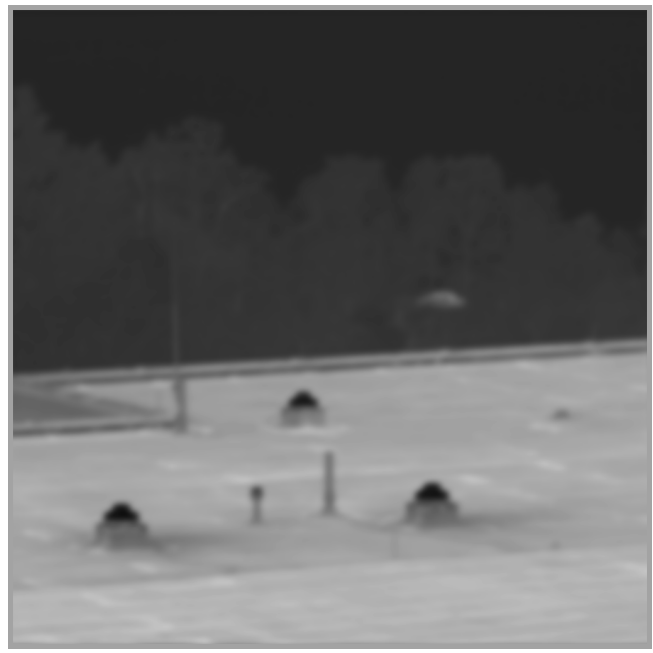

E. $\mathrm{SA}+\mathrm{Wiener}$

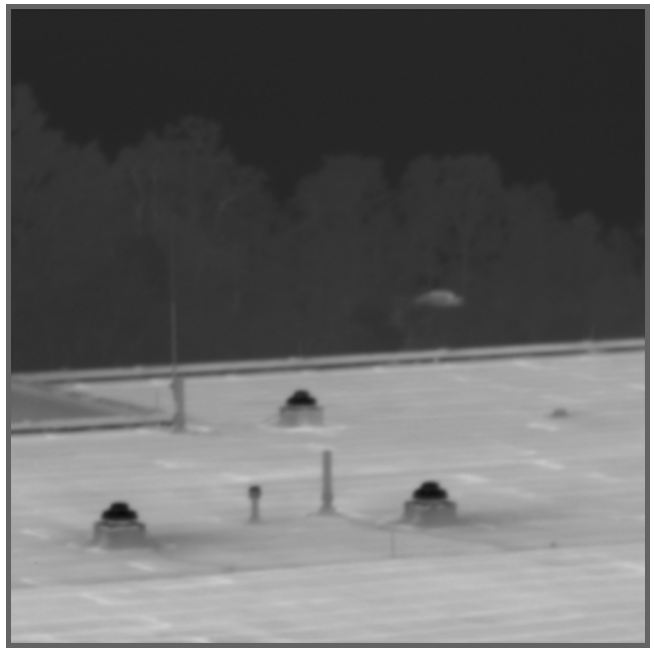

B. Cubic o-Moms

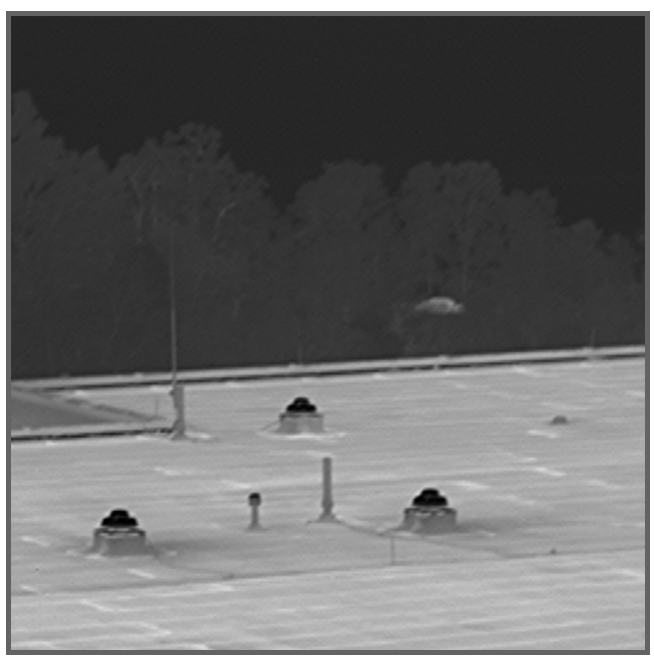

D. 2D-5PCC-R

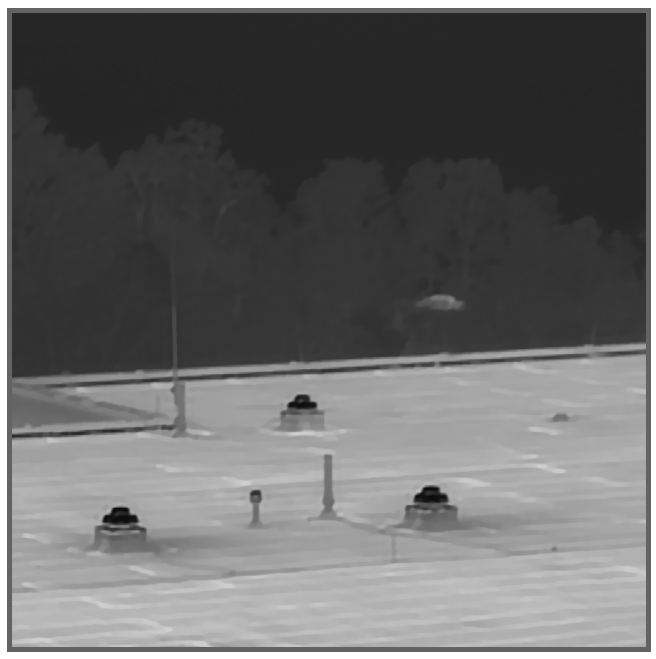

F. Norm 2 Data

Fig. 9. Super-resolution results for a microscanned infrared system. 


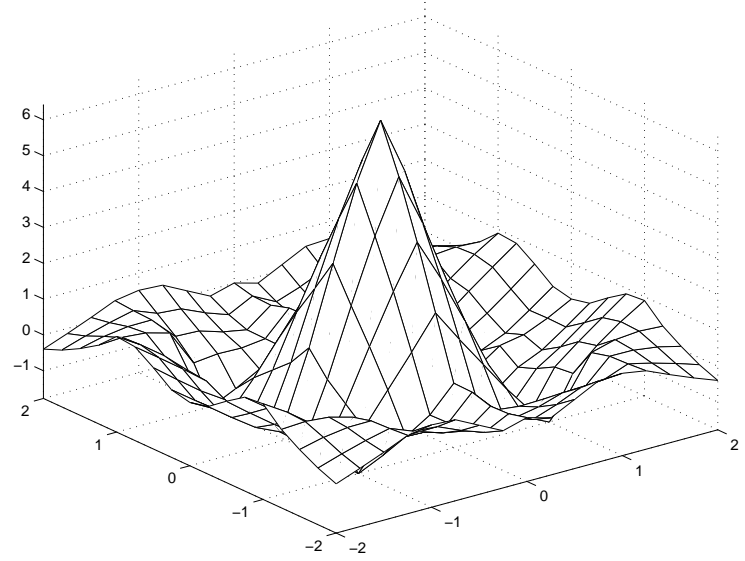

A. Small-kernel Wiener filter

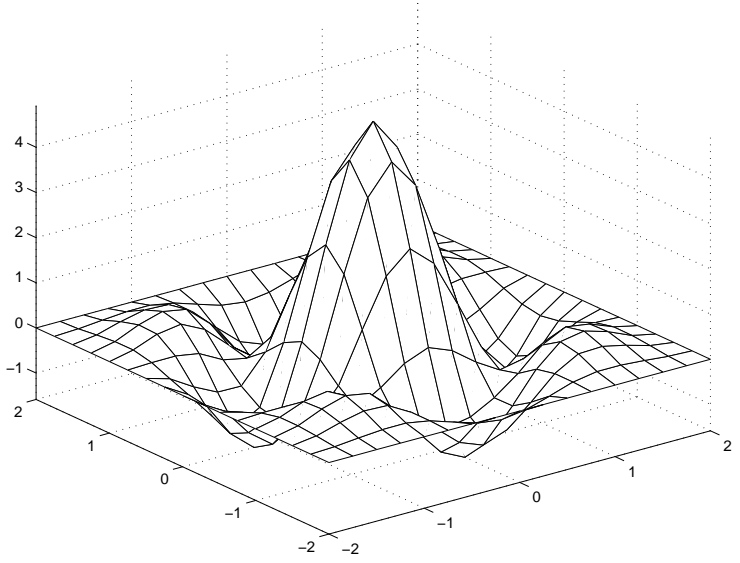

B. 2D-5PCC-R

Fig. 10. The small reconstruction and restoration kernels for the real image experiment. 